\title{
Médiévales
}

Langues, Textes, Histoire

48 | printemps 2005

Princes et princesses à la fin du Moyen Âge

\section{La cité-État italienne du Moyen Âge. Culture et liberté}

\section{Mario Ascheri}

\section{(2) OpenEdition}

1 Journals

Édition électronique

URL : https://journals.openedition.org/medievales/4403

DOI : $10.4000 /$ medievales.4403

ISSN : $1777-5892$

Éditeur

Presses universitaires de Vincennes

\section{Édition imprimée}

Date de publication : 1 juin 2005

Pagination : 149-164

ISBN : 2-84292-169-0

ISSN : 0751-2708

Référence électronique

Mario Ascheri, «La cité-État italienne du Moyen Âge. Culture et liberté », Médiévales [En ligne], 48 | printemps 2005, mis en ligne le 28 juin 2008, consulté le 22 avril 2022. URL : http://

journals.openedition.org/medievales/4403; DOI : https://doi.org/10.4000/medievales.4403

Ce document a été généré automatiquement le 22 avril 2022.

Tous droits réservés 


\title{
La cité-État italienne du Moyen Âge. Culture et liberté
}

\author{
Mario Ascheri
}

1 Le thème des cités-États italiennes au Moyen Âge est en même temps classique et très complexe, et l'on peut ajouter que ce n'est pas un thème à la mode dans notre historiographie - qui néanmoins se concentre très souvent sur l'histoire des villes.

2 Normalement on préfère parler de la ville médiévale en général, en prenant parfois l'exemple du grand tableau de l'histoire européenne réalisé par Marino Berengo, le grand maître de ces études1; ou bien les études urbaines se concentrent sur des situations locales, et nous avons alors des informations détaillées, qui aident aussi à ouvrir de nouvelles perspectives historiographiques, mais les grands panoramas restent très rares, comme ceux qui ont paru récemment en France à l'occasion du programme de l'agrégation d'Histoire 2004-20062. En France ont été publiées aussi de très bonnes monographies sur des villes (Padoue, Venise, Sienne, Arezzo, Rome, Gênes)3 ou sur des régions, comme le Latium, l'Ombrie, la Lombardie, les Marches4, mais c'est plutôt l'historiographie anglo-américaine qui a considéré la cité-État italienne spécifiquement pour ses valeurs républicaines; en particulier les historiens anglais5, peut-être plus intéressés justement parce qu'ils vivent sous des institutions monarchiques..., alors que, dans l'historiographie italienne, nous avons souvent peur d'être taxés de nationalisme quand nous soulignons les caractéristiques de notre histoire dans sa différence avec celle des autres pays ; la pudeur conseille de nuancer. En outre, aujourd'hui, si nous soulignons la grandeur des villes du centre-nord du pays, nous avons peur de servir ainsi un parti politique parfois aux limites du séparatisme, et en même temps de renforcer la fracture toujours existante entre le nord et le sud du pays.

3 Mais, mise à part la contingence politique, dans la longue durée il y a surtout comme une sorte de téléologisme. Si l'on tient compte de ce qui s'est passé après le Moyen Âge, on trouve peu de motifs qui permettent de considérer la ville médiévale italienne comme État, et comme bon gouvernement. Sur un plan général, il faut rappeler le jugement très important d'Antonio Gramsci, le grand écrivain communiste, découvert 
après la guerre par une historiographie attentive aux effets politiques immédiats de ses propres jugements. Si l'on considère une longue période - soulignait Gramsci -, ces pouvoirs urbains ont interdit la formation de l'État national en Italie, et les oligarchies qui les gouvernaient pendant l'Ancien Régime ont bloqué la société partout6. Même quand la ville avait gardé à cette époque un gouvernement républicain, elle n'était pas exonérée de critiques.

On rappelle toujours le jugement sévère de Montesquieu sur Venise et Lucques - quitte à oublier les considérations positives de ses contemporains, par exemple celles de De La Lande et de Giuseppe Lampredi sur Gênes7. On a vite fait de déstructurer les évaluations positives de certains historiens sur le passé de ces villes, comme celles de Sismondi8: on écrit généralement aujourd'hui que l'économie était dans tous les cas surtout agricole, et que le capitalisme était uniquement commercial; les institutions étaient tenues dans les meilleurs des cas par des oligarchies nobiliaires ou marchandes, mais toujours des oligarchies; la culture présentait de nombreux éléments chevaleresques, nobiliaires, même quand les gouvernements étaient dits " populaires ». L'idée dominante est qu'il y avait certes des éléments remarquables dans cette histoire urbaine, mais bien limités dans le temps et discutables dans la longue durée pour l'histoire même de l'Italie9.

L'héritage de Rome et l'affrontement avec l'empereur Frédéric Barberousse : la liberté comme consuetudo

5 Je ne veux bien sûr pas bouleverser complètement ces jugements. Je veux simplement essayer d'enrichir et mieux articuler le jugement grâce à plusieurs considérations, en particulier à propos de l'héritage de la culture romaine10.

6 En effet, je pense que la difficulté principale, quand on cherche à évaluer cette expérience-là globalement, est d'apprécier, mettre en balance, comparer les différents éléments dans le contexte de l'Europe monarchique du temps11, et surtout l'élément culturel et idéologique qui - à mon avis - était le principal et le plus fort dans la longue durée - qu'il fût d'origine romaine ou non -, et dont l'héritage a été transmis jusqu'à nos jours.

7 Mais revenons à notre sujet. Non seulement une partie de la culture romaine a survécu pendant le haut Moyen Âge, et surtout en Italie, mais les renaissances de l'Empire, carolingienne puis ottonienne, et la puissance culturelle croissante de l'Église de Rome expliquent beaucoup de notre histoire - comme en outre les liens très forts longtemps maintenus avec Byzance.

8 Les lois promulguées par les empereurs pour le Royaume d'Italie pendant les $\mathrm{x}^{\mathrm{e}}-\mathrm{XI}^{\mathrm{e}}$ siècles relèvent de la tradition du droit écrit, qui a laissé en Italie une grande partie des témoignages de cette période - par ailleurs coutumière12. Il suffit de rappeler l'exemple de l'édit sur les fiefs du Royaume d'Italie promulgué par le roi-empereur Conrad, une loi d'extraordinaire importance dans l'histoire féodale et pas seulement de l'Italie.

9 Mais on peut aussi faire des observations plus circonscrites, par exemple sur le titre de dux (plus tard doge) qu'on utilise à Venise pour indiquer le premier officier de la ville, bien avant que le titre de "consul» ne soit généralisé dans les villes du Royaume d'Italie, c'est-à-dire à partir de la fin $\mathrm{du}^{\mathrm{XI}}{ }^{\mathrm{e}}$ siècle; un titre utilisé bien avant l'apparition du mot «Commune ", au début du XII siècle, pour indiquer la personnalité 
abstraite de la nouvelle organisation qui régit la civitas, au lieu de ou en compétition avec le comes, et/ou l'évêque ou d'autres officiers13.

10 Le $\mathrm{XI}^{\mathrm{e}}$ siècle est très important pour la ville italienne, bien que les documents soient rares. On parle justement de tournant ou d'essor. Le grand débat politique et culturel sur les investitures utilise des concepts de droit romain et ce même droit est bientôt entendu comme patrimoine de tous: l'Expositio ad librum Papiensem (ouvrage très important, conservé dans un seul manuscrit napolitain), bien avant la fin du XI siècle, parle déjà du droit romain comme d'une lex generalis omnium, « loi de tous », ce qui est bien paradoxal puisqu'il s'agit d'un texte d'exégèse sur le droit lombard et carolingien 14 ! Et, quand l'empereur allemand veut connaître les droits de l'Empire sur le gouvernement de l'Italie au milieu du XII ${ }^{e}$ siècle, il s'adresse aux juristes bolonais qui enseignent le droit romain.

11 À partir de ce moment-là, la légitimité est discutée suivant des critères issus du droit romain : praescriptio, iura regalia, iurisdictio, dominium, res publica. Surtout, on parle de consuetudo, parce que les villes ont reçu des rois de larges privilèges pendant les crises dynastiques de la deuxième moitié du $\mathrm{x}^{\mathrm{e}}$ siècle, quand il fallait fortifier et résister aux invasions - comme celles des Hongrois.

12 Les privilèges sont devenus pour elles des consuetudines, que l'empereur voudrait effacer15; paradoxalement, les libertés des villes sont revendiquées en tant que coutumes, usages ; c'est-à-dire le contraire de ce que l'Église avait fait sous le pape de la réforme, Grégoire VII : car lui voulait la libertas ecclesie contre les traditions, les malae consuetudines de l'Empire et des églises locales, qui étaient au service des pouvoirs laïcs. Les villes ne vont pas changer : elles font la Ligue lombarde ou la ligue toscane (societas Tuscie) de la même manière qu'avait été établie la Commune de Gênes, c'est-à-dire comme une entreprise commerciale plutôt que comme un foedus, une fédération qui donnerait vie à un sujet politique nouveau, permanent, à l'État au-dessus des Communes membres. Le fédéralisme n'existe pas dans notre tradition médiévale.

Dans tous les cas, la libertas est l'objet des revendications des Communes, qui utilisent des formules typiques de la tradition républicaine romaine - au moment où Milan, un peu avant Padoue, commence à se penser comme une secunda Roma - comme d'autres le feront plus tard, Sienne et Florence par exemple16.

Otton de Freising17 - le savant évêque oncle de l'empereur Frédéric I ${ }^{\mathrm{er}}$ - écrit que les villes italiennes, «jardins des délices », vivent dans l'imitation de la sagesse ancienne, de la sollertia antiquorum Romanorum, « du savoir-faire des Romains antiques », en ce qui concerne la gestion des affaires publiques; elle sont sous le gouvernement des consuls au lieu de celui des rois, des consuls qui changent chaque année afin de freiner le désir de pouvoir. Mais Otton doit faire aussi des critiques, parce que les Communes déclarent qu'elles vivent selon le droit, alors qu'au contraire, à son avis, elles violent le droit celui de l'Empire naturellement.

15 Et en effet Boncompagno de Signa, le grand rhétoricien enseignant à Bologne au début du XIII ${ }^{\mathrm{e}}$ siècle, écrit que les consuls obéissent aux statuts de la ville non obstante aliqua lege que contra statutum dicere videatur, "bien que certaines lois [de l'Empire] semblent s'opposer au statut $» 18$.

16 Pendant le siège de Milan, écrit Rahewin, le chroniqueur successeur d'Otton, plusieurs citoyens, au lieu de se rendre, préférèrent mourir pro libertate patriae et honore civitatis : les valeurs - disaient-ils - qu'appréciaient déjà leur pères et leurs ancêtres. 
17 La formule du Digeste, libertas inestimabilis res est (Dig. 50.17.106), est rappelée dans la même source (Rahewin), qui vient de l'Empire! Même un chroniqueur indépendant comme l'archevêque Romuald de Salerne écrit que l'ambassadeur de la Ligue lombarde parlait des libertés héritées par ses bisaïeux, aïeux et pères, qu'il faudrait maintenant sauvegarder pour sauver l'honneur de l'Italie; l'ambassadeur était comme les autres Lombards19: tous versés in utraque militia, c'est-à-dire «courageux au combat et admirablement formés à haranguer le peuple » (in bello strenui et ad contionandum populo mirabiliter eruditi). Et ici se trouve mis en lumière un autre caractère typique de la libre vie politique de ces villes : l'éloquence civile, parfaitement cultivée et appréciée20.

Dans ce contexte on comprendra que la ville de Rome, quand elle s'organise en Commune, restaure avant tout le Sénat: la renovatio Senatus, le désir de couronner Frédéric et la tentative malheureuse d'Arnaud de Brescia suivent les schémas de l'héritage romain21. Pise, qui a des rapports très étroits avec le monde byzantin et où travaille alors un homme aussi savant que le juge et traducteur Burgundio, rédigea au milieu du XII siècle un constitutum legis fortement inspiré du droit romain22; elle a aussi des iuris periti, « spécialistes du droit », dans ses cours judiciaires. À Venise comme à Pise, toujours au milieu du siècle, des documents donnent les premiers noms de jurisconsultes - à partir de Gratien, le rédacteur du Décret. Ils deviennent très fréquents à partir de la fin du XII ${ }^{\mathrm{e}}$ siècle, comme autant de consultants qui donnent des responsa comme leur anciens collègues romains; c'est ainsi que les doctrines romanistes des universités s'introduisent dans la pratique judiciaire de tous les jours23. Le mythe de Rome est très efficace: depuis longtemps chaque ville était une civitas, comme Rome, mais maintenant seulement, ses habitants sont dits comme autrefois cives, « citoyens ». Par exemple, on parlait encore génériquement des homines de Gênes au moment où ils recevaient une donation en Orient au début du XII ${ }^{e}$ siècle, mais plus tard ils deviennent des « citoyens » de l'universitas civitatis, ou Commune civitatis24.

La ville avait déjà une forte identité grâce aux privilèges royaux. La menace de l'empereur Barberousse de mettre fin aux autonomies locales souligne encore un esprit de liberté déjà fort: Otton de Freising est très clair à ce sujet, comme Romuald de Salerne.

21 L'intervention de Frédéric a commencé à révéler l'Empire comme facteur de déséquilibre et de complication de la réalité politique25, et non plus comme un élément de renforcement des structures publiques et de maintien de la paix. La ligue lombarde souligne la personnalité politique des différentes villes, et l'armée de chaque ville renforce la conscience de son indépendance, de son identité et de sa capacité à gouverner par elle-même!

Fédéralisme et citoyenneté

22 L'exemple de Rome est toujours présent et, comme Rome, les grandes villes aspirent à élargir leur propre espace. Les alliances comme la ligue lombarde (qui dura seulement quelques années) sont seulement des accords militaires valables jusqu'à la fin du péril : passé le péril, chaque ville commence à rechercher de nouveaux espaces économiques et politiques, et c'est alors le temps de la guerre et de la défaite pour beaucoup de centres voisins.

23 L'honor civitatis impose de grandir, même par la violence militaire, et les grandes victoires romaines n'avaient pas besoin d'être soulignées. Mais surtout les textes romains préservent la mémoire du temps où le populus avait tout le pouvoir, avant la lex 
regia, et où les juristes débattaient sur le caractère définitif ou non de cette loi : peut-on se défaire de sa propre souveraineté ? L'empereur Constantin s'était trompé sur ce point-là et sa donation était nulle du point de vue juridique. Le juriste de Bologne Azon, autour de l'an 1200, a des réflexions très intéressantes sur ces points 26.

Il faut aussi considérer la paix de Constance de 1183 : l'empereur a fait des concessions très importantes aux Communes de la ligue lombarde, mais, ce faisant, n'a-t-il donc pas lui-même privé l'Empire de ses droits? La pratique dira bientôt que même les autres Communes, hors de la ligue, jouissent des mêmes privilèges; naturellement, la révocation de la paix par Frédéric II sera sans effets. Quelques années auparavant, Boncompagno pouvait écrire que «puisque seule l'Italie, parmi toutes les provinces du monde, jouit d'un privilège spécial de liberté, il faut honorer particulièrement les Italiens, et toutes les provinces du monde sont à juste titre tenues de leur être soumises "27. Et ce n'est pas tout. On sait, en effet, que les Lombards sont « maîtres de leur liberté, excellents défenseurs de leur propre droit, ayant le plus souvent combattu pour leur liberté, à juste titre sénateurs d'Italie »28.

Le droit romain peut être évoqué aussi en faveur des Communes, car « comme les lois impériales le proclament, l'Italie n'est tributaire de personne mais elle est maitresse des provinces » 29 ; dans le projet de l'empereur, l'Italie, « de souveraine des provinces, devenait tributaire $» 30$; mais, poursuit Boncompagno, «nous avons entendu des anciens et nos pères nous ont dit qu'ils ne nous laissaient pas d'or mais des armes, afin que nous défendions la liberté de la patrie. Notre or, en effet, c'est la liberté »31.

Les villes ont appris que la reconnaissance des droits par l'Empire peut être utile ; mais les concessions ne sont pas nécessaires : ce qui est vraiment nécessaire c'est l'armée et un pouvoir politique très fort dans la ville, de préférence fondé sur le consentement le plus large des citoyens.

27 Car la ville est très sensible aux assemblées publiques - harangues, conciones, conventus - et aux problèmes de la religiosité locale : elle stimule le culte des saints locaux, elle construit la cathédrale32, elle organise de grandes cérémonies publiques et dépense beaucoup d'argent pour la construction de grands palais publics pourvus de fresques politiques. Être ou non citoyen devient très important: afin de jouir des privilèges conquis par la ville, mais aussi pour le payement des impôts, pour le service militaire, pour la protection judiciaire, etc. L'homme de la ville est avant tout « citoyen »33, civis - Florentinus ou Mediolanensis ou Ianuensis... : inutile donc de chercher une date de mort du Royaume d'Italie.

À ce stade surgissent les problèmes du droit international privé : chaque ville a son propre droit, mais alors, comment considérer les citoyens de l'autre ville? Et la multitude des relations juridiques nouées entre citoyens de différentes villes, c'est-àdire les mariages mixtes, les sociétés commerciales, les héritages comprenant des biens situés dans différentes villes...?

Volonté politique et loi : le XIII ${ }^{\mathrm{e}}$ siècle

Boncompagno, comme d'habitude, est très clair sur ce point : le ius civile (droit civil) ne régit pas seulement une petite pars terrarum; désormais les statuts sont importants, et ils sont l'expression «de la libre volonté de ceux qui décident» (arbitrium constituentium), de la volonté purement politique34.

Cette volonté fait des lois, et il faut faire attention! « Il n'y aura pas liberté de juger des lois mais il faudra juger suivant les lois, parce que, quand elles auront été décidées et 
édictées, le juge ne pourra plus les juger mais il devra juger en fonction d'elles »35; c'est ce qu'écrivait vers 1222-1242 l'auteur inconnu de l'Oculus pastoralis. Cependant, les lois, poursuit l'Oculus, sont le fruit des assemblées, réunies dans les formes dictées par la consuetudo patrie; et elles sont généralement justes, parce qu'il faut rappeler que «ce qui est demandé par le plus grand nombre est obtenu et la vérité est reconnue par les plus grands hommes, de même que le savoir civique est clairement reconnu »36.

31 Tout ceci, comme les autres écrits du premier xIII siècle, a contribué à répandre la culture de la légalité et de l'éloquence civile, grâce aux ouvrages de Cicéron et à son enseignement républicain37, en particulier dans le De officiis et le De inventione, de même que, à partir du Bellum Cathilinae de Salluste, on établit la liaison entre conditions de liberté et richesse de la ville. Des ouvrages au service de la ville-État sont écrits, pour construire le monde de valeurs collectives des cités, et ce n'est pas un hasard si, généralement, leurs auteurs ne sont pas des professeurs d'université mais des hommes qui travaillent en relation avec les chancelleries communales 38.

L'Empire, théoriquement, n'est pas effacé, mais Frédéric II a trop de prétentions aux yeux des villes habituées à se gérer par elles-mêmes. À Bologne on va jusqu'à inventer un faux privilège de Théodose afin de donner à l'université un passé romain, de lui attribuer un plus grand prestige et de la rendre indépendante des pouvoirs prétendûment universels du temps39. À cette date-là les juristes ont déjà accepté pleinement la réalité de la législation communale; mais on parle des statuts avec des nuances diverses. Certains pensent qu'ils représentent le ius civile de la ville comme c'était le cas du corpus iuris pour les Romains; mais d'autres pensent qu'ils correspondent seulement aux édits du praetor, et ne sont qu'une partie seulement du droit civil de la ville, qui doit être complété par le droit commun universitaire.

Une certaine ambiguïté est liée à l'évolution de différentes réalités. Ainsi, les consuls du $\mathrm{XII}^{\mathrm{e}}$ siècle étaient des citoyens du lieu; or, au $\mathrm{XIII}^{\mathrm{e}}$ siècle, le rector civitatis est un étranger, investi d'une locatio operarum. Reste le modèle républicain romain de la temporalité de l'office, qui dure généralement un an 40 - tandis que la flamboyante vie urbaine stimule même la renaissance des leges sumptuariae, les lois somptuaires déjà inscrites dans la tradition romaine 41.

En même temps, au milieu du siècle, la mort de Frédéric II en 1250 est une libération pour les villes, même les villes gibelines. Elles ont désormais compris que les empereurs ne sont pas tous les mêmes. Confrontées à la vacance durable de l'office impérial résultant des conflits pour l'élection, les villes en arrivent à intervenir dans l'élection $\mathrm{du}$ nouvel empereur en tant que forces politiques constitutionnelles, c'est-à-dire comme des membres de la constitution matérielle de l'Empire. Et, ce faisant elles parlent de negotiorum gestio (" gestion des affaires »), suivant encore une fois le schéma romain 42 .

Nous sommes alors à peu près au milieu du xiII siècle, quand - après la mort de Frédéric II - le phénomène communal est arrivé à maturité. C'est-à-dire qu'il arrive à un tournant précis : certaines villes restent indépendantes mais connaissent de graves crises politiques; elles sont amenées à recourir à des seigneurs qui sont généralement des nobles, militaires, comme il arrive dès la première moitié du xIII siècle dans certaines villes $d u$ nord-est - Trévise, Vérone, etc.43. Mais d'autres villes restent indépendantes et sans seigneur malgré leurs évidentes difficultés politiques; cette situation extraordinaire requit la création d'une idéologie complexe - qui s'édifia en s'appuyant sur des légendes, qui associaient toujours la fondation de la ville à Rome, ou 
à Énée, ou qui situaient la ville sur un lieu sacré dédié à un dieu antique, Mars pour Florence par exemple.

Le tournant du milieu du Duecento :

le nouveau "popolo » contre les " puissants" 
familles, imposant toujours des règles d'incompatibilité - une tradition quasiment perdue dans notre République...

De plus, la justice des Communes est bien différente de celle des rois, qui ont des lois cachées, in pectore recondite : ici, dans les villes, « les recteurs sont soumis aux lois; le peuple est jugé seulement suivant les lois de sa cité »56, et «([les juges] n'osaient pas introduire la moindre nouveauté par rapport à la loi écrite) »57; un peu comme chez les Romains qui « créèrent l'assemblée, laquelle chaque jour réunissait trois cent vingt conseillers... pour traiter les problèmes dignes d'attention $» 58$.

Mais le plus étonnant pour nous aujourd'hui est que dans les Communes " populaires ", les magnats sont soumis à des règles négatives, inégales ou pour mieux dire des règles qu'on appellerait aujourd'hui discriminatoires, puisqu'elles violent le principe de l'égalité juridique formelle des citoyens, pour mieux défendre (en principe) une possibilité d'égalité réelle59.

Les hommes définis comme puissants (magnates) ne sont pas éligibles aux offices les plus importants de la ville et ils sont punis avec une plus grande sévérité, ou encore ils sont solidairement responsables avec les autres membres de leur groupe en cas de violation de la loi. À l'intérieur de la Commune les institutions dites du «Peuple » deviennent comme un État dans l'État. Le «Peuple 60 a ses offices, à commencer par le capitaine du peuple, un citoyen d'abord (mais par la suite un étranger) qui contrôle le podestat, recteur de la ville; ce dernier est, comme on l'a dit plus haut, l'officier qui a remplacé les consuls et est officiellement responsable de la gestion de la ville, suivant les règles édictées dans les statuts et réformées chaque année suivant le modèle de l'édit du praetor romain. Le podestat amène dans la ville ses juges, qui siègent a latere et sont nommés assessores, comme chez les Romains ; de plus, comme chez les Anciens, ils sont chargés de la répression criminelle publique, mais ce qui introduit une nouveauté de taille par rapport à la tradition accusatoire antérieure, c'est qu'ils le sont ex officio, per inquisitionem61.

Le capitaine pour sa part est une sorte de tribunus plebis qui a gagné dans la compétition politique et sociale : le statut du Peuple a créé une institution privilégiée dans la ville, qui contrôle la Commune traditionnelle. Avec d'incroyables complications juridiques et une duplication des offices, la vieille Commune de tous les citoyens reste, mais le parti du Peuple, l'organisation d'une partie seulement de la société, contrôle, un peu comme un Soviet, toute l'organisation publique.

En disant cela, je me permets de généraliser et de simplifier des processus qui se sont réalisés différemment suivant les villes; dans tous les cas, comme à Rome, il n'y a pas seulement deux catégories sociales, et le peuple médiéval n'est pas la plebs, la catégorie où étaient relégués les niveaux les plus humbles de la société, sa fraction la plus turbulente.

La société urbaine médiévale n'est pas un paradis ; au contraire, étant très sectaire et compétitive, elle produit de nombreux exclus62, mais elle permet aussi de grands progrès, les «sùbiti guadagni » de Dante, qui plusieurs fois exalte la liberté ; surtout elle libéralise la force de travail, en favorisant l'affranchissement des serfs dans les campagnes63, non sans exalter le droit romain comme dans la loi florentine, et en facilitant l'immigration en ville des paysans et par là l'enrichissement général.

Le développement de la pensée républicaine 
de Machiavel - loués plus tard par Rousseau - sont en quelque sorte un traité de droit constitutionnel comparé, des anciens et des modernes, qui a transmis en Europe la pensée républicaine des villes italiennes69. L'auteur, rapidement condamné par l'Église romaine et apparemment oublié par les écrivains de la "raison d'État », a cependant introduit dans notre tradition occidentale la culture politique de ces villes; on le comprend bien quand il écrit par exemple que « les républiques où la vie politique s'est maintenue sans se corrompre ne tolèrent pas qu'un seul de leurs citoyens soit noble ou mène une vie noble, ... c'est-à-dire qu'il vive dans l'oisiveté du profit de ses biens »70.

On a durant cette période âprement débattu sur la manière de contenir le pouvoir des puissants, de partager équitablement le gouvernement, de le définir par la loi, de l'ouvrir à plusieurs catégories sociales mises en condition d'égalité. Au point que Machiavel, se prononçant contre une longue tradition, en vient même à considérer comme positifs les débats politiques voire les conflits qui divisaient la ville. Mais malheureusement la grandeur de cette pensée n'avait plus place dans la pratique politique, et Guicciardini - se référant sans le dire à Machiavel - observait que ceux qui ont toujours voulu imiter les Romains sont dans l'erreur, parce que, pour ce faire, il 
faudrait avoir leurs institutions; or désormais les institutions communales avaient changé et elles étaient en crise depuis longtemps71.

Pour conclure

La fin des libertés et la décadence oligarchique et nobiliaire d'Ancien régime ont terni cette réalité. Mais il est temps de la reconnaître sans peur. L'historiographie se concentre parfois sur mille détails et oublie les grands problèmes et les réalisations du passé. Au contraire, il faut les rappeler en soulignant que - sous tous les gouvernements - les conquêtes civiles ne sont jamais définitives, à commencer par la démocratie même, que les solutions apportées aux problèmes de l'équilibre des pouvoirs ne sont jamais définitives non plus, parce qu'elles ne sont jamais parfaites. Il n'y a pas de progrès continu et uniforme - comme l'a montré très clairement le $\mathrm{xx}^{\mathrm{e}}$ siècle, le pire peut-être dans l'histoire de l'Europe.

Les villes n'ont pas laissé seulement de beaux monuments ou des fresques; les citésÉtats surtout ont laissé des idées; des idées qui ont illuminé certains tournants très délicats de l'histoire occidentale : après les jugements de Brunetto Latini et de Bartolo de Sassoferrato, la synthèse de Machiavel ne valait pas seulement pour Florence, mais devait inspirer, en accord avec le souvenir de la Rome antique, tous les mouvements républicains de la période moderne, des Pays-Bas à l'Angleterre révolutionnaire du XVII ${ }^{\mathrm{e}}$ siècle, jusqu'aux grandes révolutions américaine et française72. Les Romains avaient beaucoup parlé de liberté naturelle des hommes, les villes populaires italiennes proclamèrent la liberté et l'égalité pour la population urbaine, et les Révolutions des $\mathrm{XVIII}^{\mathrm{e}}$-XIX ${ }^{\mathrm{e}}$ siècles les étendirent à tous les hommes. Il y a une certaine continuité. Pour cette raison aussi, il est juste de ne pas oublier aujourd'hui ces institutions de liberté.

\section{NOTES}

\section{\#Notes}

1. M. BERENGo, L'Europa delle città, Turin, 1999. Voir aussi F. BocchI, M. GHIZZONI, R. SMURA, Storia delle città italiane. Dal Tardo antico al primo Rinascimento, Turin, 2002, et encore Modelli di città. Strutture e funzioni politiche, P. Rossi éd., Turin, 1987.

2. Quelques exemples: F. FRANCESCHI, I. TADDEI, Les villes d'Italie du milieu du XII siècle au milieu $d u$ XIV e siècle, Rosny-sous-Bois, 2004; P. BOUCHERON, Les villes d'Italie (vers 1150-vers 1340). Historiographie. Bibliographie. Enjeux; N. Bouloux, Les villes d'Italie du milieu du XII siècle au milieu du XIV siècle. Économies, sociétés, pouvoirs, cultures; P. GILLI, Villes et sociétés urbaines en Italie, milieu XII milieu XIV siècle; particulièrement utile le volume historiographique de F. MENANT, Les villes italiennes, XII ${ }^{e}-\mathrm{XIV} \mathrm{V}^{e}$ siècle. Enjeux historiographiques, méthodologie, bibliographie commentée, ouvrages tous parus en 2004 à Paris.

3. Voir les ouvrages de Jacques Heers, Pierre Racine, Odile Redon, Gérard Rippe, Élisabeth Crouzet-Pavan, Jean-Pierre Delumeau, Étienne Hubert.

4. Voir les travaux de Pierre Toubert, Jean-Claude Maire Vigueur, François Menant, Philippe Jansen. De J.-Cl. Maire Vigueur, citons aussi le récent Cavaliers et citoyens. Guerre, conflits et société 
dans l'Italie communale, XII ${ }^{e}-X I I I^{e}$ siècles, Paris, 2003, qui propose une nouvelle lecture de l'historiographie communale.

5. Voir la synthèse de D. WALEY, en français : Les Républiques médiévales italiennes, Paris, 1980, et le grand livre de Ph. JONES, The Italian City-State. From Commune to Signoria, Oxford, 1997. Intéressante synthèse aussi de N. RUBINSTEIN, «Le origini medievali del pensiero politico repubblicano dei secoli XV e XVI ", dans Politica e cultura nelle Repubbliche italiane dal Medioevo all'età moderna : Firenze - Genova - Lucca - Siena - Venezia, S. AdoRni-BRACCESI, M. Ascheri éd., Rome, 2001, p. 1-20.

6. Voir la discussion dans M. VIroLI, "Le ragioni di un dibattito", dans Politica e cultura nelle Repubbliche..., op. cit., p. 335-346.

7. Pour Gênes, voir désormais les considérations de R. SAVELLI, « Scrivere lo statuto, amministrare la giustizia, organizzare il territorio », dans Repertorio degli statuti della Liguria (secc. XII-XVIII), R. SAVELLI éd., Gênes, 2003, p. 3-191.

8. Cf. J. C. L. S. SIsmondi, Storia delle Repubbliche italiane, prés. P. SCHIERA, Turin, 1996.

9. J'ai examiné ces tendances dans M. AschERI, «Les cités-États et les Communes: quelques problèmes historiographiques", dans Scrivere il Medioevo. Lo spazio, la santità, il cibo, Un libro dedicato ad Odile Redon, B. LAURIOUX et L. MOULINIER-BROGI dir., Rome, 2001, p. 55-75.

10. Voir les contributions du volume Roma antica nel Medioevo. Mito, rappresentazioni, sopravvivenze nella «Respublica Christiana » dei secoli IX-XIII, Milan, 2001.

11. Cf. Gewalt und ihre Legitimation im Mittelalter, G. MENSCHING éd., Würzburg, 2003.

12. Voir F. BOUGARD, La justice dans le Royame d'Italie: de la fin du VIII e siècle au début du XI siècle, Rome, 1995.

13. O. BANTI, " "Civitas" e "Commune" nelle fonti italiane dei secoli XI e XII ", dans Forme di potere e struttura sociale in Italia nel Medioevo, G. RosSETTI éd., Bologne, 1977, p. 217-232.

14. Sur les sources juridiques du temps, l'exposé le plus récent est celui de M. Ascheri, I diritti del Medioevo italiano (secoli XI-XV), Rome, 2000.

15. Sur ce point il existe une abondante littérature, que j'ai présentée dans M. AsCHERI, « Statuti e consuetudini tra storia e storiografia ", dans Signori, regimi signorili e statuti nel tardo Medioevo, R. Dondarini, G. M. VARAnini, M. Venticelli éd., Bologne, 2003, p. 21-31.

16. Les sources qui suivent sont rappelées brièvement dans le riche article de J. H. MunDY, « In Praise of Italy: The Italian Republics", Speculum, 64, 1989, p. 815-834 (un article ignoré de l'historiographie italienne), et par son précurseur R. L. BENSON, «Libertas in Italy (1152-1226) », dans La notion de liberté au Moyen Âge: Islam, Byzance, Occident, G. MAKDISI, D. SOURDEL, J. SOURDELTHOMine éd., Paris, 1985, p. 191-213.

17. Source fondamentale : OtTONIS EPISCOPI FrISIGENSIS et RAWEGINII Gesta Federici, F.-G. SCHMALE éd., Darmstadt, 1965 ; bonne analyse dans R. L. BENSON, « Libertas... », loc. cit., p. 193-196.

18. Parmi les nombreuses œuvres de Boncompagno, voir sur ce point le Cedrus, dans L. RocKINGER, Briefsteller und Formelbuechern des elften bis vierzehnten Jahrhunderts, I, Munich, 1863, p. 121-127. Sur l'auteur, bonnes analyses d'Enrico Artifoni, particulièrement «Retorica e organizzazione del linguaggio politico ", dans Le forme della propaganda politica nel Due e nel Trecento, P. CAMMAROSANO éd., Rome, 1994, p. 157-182; voir aussi les autres contributions de ce volume et les actes du colloque Il pensiero e l'opera di Boncompagno da Signa, M. BALDINI éd., Greve in Chianti, 2002.

19. Son Chronicon figure dans Rerum Italicarum Scriptores, VII 1, éd. C. A. GARUFI, Bologne, 1909-1935, p.271-275 ; les textes sont examinés dans R. L. BENSON, "Libertas... ", loc. cit., p. 196-197. Voir aussi Romualdo II Guarna, Chronicon, éd. C. BonetTi, Cava de' Tirreni, 2001.

20. Voir encore E. ARTIFONI, «Gli uomini dell'assemblea. L'oratoria civile, i concionatori e i predicatori nella società comunale ", dans La predicazione dei frati dalla metà del '200 alla fine del '300, Spolète, 1995, p. 147-188. 
21. J.-Cl. Maire Vigueur, « Il Comune romano », dans Roma medievale, A. VAuchez éd., Rome-Bari, 2001, p. 117-157.

22. I costituti della legge e dell'uso di Pisa (sec. XII). Edizione critica integrale del testo tradito dal «Codice Yale» (ms. Beinecke Library 415), étude introductive, texte et appendice, P. VIGNOLI éd., Rome, 2003.

23. Cf. M. AsCHERI, «I consilia dei giuristi come acta giudiziari », dans La diplomatica dei documenti giudiziari (dai placiti agli acta - secc. XII-XV), G. NICOLAI éd., Rome, 2004, p. 309-328.

24. On suit bien ces développements linguistiques dans les libri iurium : voir par exemple l'espace que leur consacre G. MilanI, I Comuni italiani, Rome-Bari, 2004, p. 75 sq.; une autre synthèse récente de E. OcchIPINTI, L'Italia dei Comuni. Secoli XI-XIII, Rome, 2000. Voir aussi M. AscherI, Istituzioni medievali, Bologne, 1999, qui consacre une grande place aux Communes.

25. Toujours important pour la question: G. TABACCO, Egemonie sociali e strutture del potere nel Medioevo italiano, Turin, 1979.

26. Cf. E. CORTESE, Il problema della sovranità nel pensiero giuridico medievale, Rome, 1982.

27. «Cum sola Italia inter cunctas mundi provincias speciali gaudet privilegio libertatis... specialius est Italicis deferendum... illis universae provincie orbis merito subesse tenentur ». La Rhetorica novissima de Boncompagno est très riche d'indications pour notre propos; elle est publiée dans Scripta anecdota glossatorum, A. GAUDENZI éd., Bologne, 1892; voir R. L. B ENSON, «Libertas... », loc. cit., p. 200-207. Sur la paix de Constance, voir M. AsCHERI, La pace di Costanza : da Odofredo a Baldo ed oltre, sous presse, dans les actes du Colloque de Pérouse, pour le $600^{\mathrm{e}}$ anniversaire de la mort de Baldo degli Ubaldi.

28. «Libertatis patroni, proprii iuris egregii defensores, ut illi qui pro libertate tuenda sepius pugnaverunt, merito sunt Italie senatores ».

29. «Sicut imperatorum leges proclamant, Italia non est tributaria, nec est provincia set domina provinciarum ».

30. « Princeps provinciarum facta est sub tributo ».

31. « $\mathrm{Ab}$ antiquis audivimus et nobis patres nostri dixerunt quod non relinquebant nobis aurum set arma, quibus nos libertatem patrie tueamur. Aurum siquidem nostrum est libertas... ».

32. A. BENVENUTI, Pastori di popolo. Storie e leggende di vescovi e di città nell'Italia medievale, Florence, 1988 ; P. BOUCHERON, "À qui appartient la cathédrale? La fabrique et la cité dans l'Italie médiévale ", dans P. Boucheron, J. CHIfFolEAu dir., Religion et société urbaine au Moyen Âge. Études offertes à Jean-Louis Biget par ses élèves, Paris, 2000, p. 95-117.

33. Voir par exemple les études réunies dans Legislazione e prassi istituzionale nell'Europa medievale. Tradizioni normative, ordinamenti, circolazione mercantile (secoli XI-XV), G. RosSETTI éd., Naples, 2001.

34. Pour de plus amples développements, voir M. AscheRI, « Il "dottore” e lo statuto : una difesa interessata », Rivista di storia del diritto italiano, 69, 1996, p. 95-113.

35. « Non erit liberum iudicare de ipsis, sed oportebit iudicare secundum eas [...] », « cum fuerint constitute et firmate, non licebit iudici de illis, sed secundum ipsas, postea iudicare » .

36. "Quod a pluribus queritur, invenitur, et veritas per ampliores homines manifestissime revelatur, sicut dicta civilis sapientia apertissime protestatur ». L'Oculus pastoralis pascens officia et continens radium dulcibus pomis suis, écrit au milieu du siècle, a été réédité par D. FRANCESCHI, dans Memorie dell'Accademia delle scienze di Torino. Classe di Scienze morali, storiche e filologiche, s. IV, 11, 1966, p. 3-70.

37. Voir Q. SKINNER, Le origini del pensiero politico moderno, I-II, Bologne, 1989 (traduction de l'édition parue à Cambridge en 1978).

38. L. BAIETTO, «Scrittura e politica. Il sistema documentario dei Comuni piemontesi nella prima metà del secolo XIII ", Bollettino storico-bibliografico subalpino, 98, 2000, p. 105-164. Fondamental sur le sujet : P. TORELl, Studi e ricerche di diplomatica comunale, Rome, 1988 (traduction de l'édition de Mantoue, 1911). 
39. A. I. PINI, « Federico II, lo Studio di Bologna e il "falso Teodosiano" », dans Deputazione di storia patria per le province di Romagna. Documenti e studi, 27, 1996, p. 27-60.

40. Voir I podestà dell'Italia comunale, I-II, J.-Cl. MAIRE VigueUR dir., Rome, 2000 ; M. V ALLERANI, «L'affermazione del sistema podestarile», dans Storia d'Italia, G. GALAsso éd., Turin, 1998, p. 385-426.

41. Disciplinare il lusso. La legislazione suntuaria in Italia e in Europa tra Medioevo ed Età moderna, M. G. MuZzarelli et A. CAMPANini éd., Rome, 2003.

42. G. DE VERGOTTINI, Il diritto pubblico italiano nei secoli XII-XV, rééd. de la $3^{\mathrm{e}}$ éd. par C. DoLCINI, Milan, 1993, p. 147 sq.

43. A. CASTAGnetTI, La marca veronese-trevigiana, Turin, 1986 ; G. M. VARANINI, "Aristocrazie e potere nell'Italia centro-settentrionale dalla crisi comunale alle guerre d'Italia », dans R. BORDONE, G. CAStelnuovo, G. M. VARANinI, Le aristocrazie dai signori rurali al patriziato, Rome-Bari, 2004, p. 121-187.

44. Voir le volume Magnati e popolani nell'Italia comunale, Actes du congrès de Pistoia (1995), Pistoia, 1997.

45. M. TURCHETTI, Tyrannie et tyrannicide de l'Antiquité à nos jours, Paris, 2001.

46. Sur son De regimine civitatis écrit en 1263 quand il était juge du podestat de Florence, voir par exemple N. RUBINSTEIN, « Le origini », loc. cit., p. 2 et 10.

47. Ptolémée est le continuateur du De regimine principum de Thomas d'Aquin, éd. R. M. SPIAzzI dans ThomAs Aquinas, Opuscula philosophica, Turin, 1954 ; le passage cité est dans THOMAS AQUINAS, Opera omnia, Paris, 1875, XXVII, col. 396a.

48. «Quando per successionem presunt, male regitur respublica... quamdiu Romani illos qui preessent elegerunt, sapientissimos elegerunt » (J. H. MUNDY, «In Praise of Italy... », loc. cit., p. 822).

49. «Apud sapientes et homines virtuosos, ut fuerunt antiqui Romani, ... regimen politicum melius fuit ».

50. «Secundum merita unicuique civi vicissim distribuuntur honores, ut antiqui fecerunt Romani ».

51. Voir Li livres dou Tresor, II, 44, F. J. CARMODy éd., Berkeley-Los Angeles, 1948, p. 211.

52. Ainsi dans le souvenir du chroniqueur florentin Giovanni Villani ( $\dagger 1348)$. Sur ces auteurs voir surtout Q. SKINNER, "Machiavelli's Discorsi and the Pre-humanist Origins of Republican Ideas ", dans Machiavelli and Republicanism, Q. SKINNER et alii éd., Cambridge, 1990, p. 121-141.

53. « Expedit regi per paucos, hoc est per divites et bonos homines ».

54. « Aucta civitate Romana facti sunt senatores, eisque data est omnis potestas, sic enim regitur civitas Venetiarum, sic civitas Florentina ».

55. Les passages cités sont extraits du De regimine civitatis (dans D. QUAGLIONI éd., Politica e diritto nel Trecento italiano, Florence, 1983, p. 349-372).

56. «Legibus astringuntur rectores politici », « solis legibus sue civitatis populum iudicat ».

57. « Non audiebant aliquam facere novitatem preter legem conscriptam».

58. «Curiam fecerunt, et quod cotidie consulebant trecentos viginti... ut que digna sunt gerant». Voir J. H. Mundy, « In Praise... », loc. cit., p. 825.

59. G. FASOLI, «Ricerche sulla legislazione antimagnatizia nei Comuni dell'alta e media Italia », Rivista di storia del diritto italiano, 13, 1939, p. 86-133, 240-309.

60. La littérature sur ce point est très vaste, voir par exemple J. KoENIG, Il « popolo » dell'Italia del nord nel XIII secolo, Bologne, 1986.

61. Bibliographie dans M. AsCHERI, I diritti..., op. cit., p. 349 sq.

62. Voir G. MILANI, L'esclusione dal Comune. Conflitti e bandi politici a Bologna e in altre città italiane tra XII e XIV secolo, Rome, 2003.

63. M. GIANSANTE, Retorica e politica nel Duecento. I notai bolognesi e l'ideologia comunale, Rome, 1999. 
64. Voir la discussion entre Q. Skinner et M. Donato dans Politica e cultura nelle Repubbliche..., op. cit., p. 21-79. Sur le contexte, avec des précisions sur la culture juridico-institutionnelle, voir M. AsCHERI, Siena e la città-Stato del Medioevo italiano, Sienne, 2004.

65. On trouve en effet: Appius Claudius Cecus, Attilius Regulus, les deux Brutus, Cato Uticensis, Lucius Cecilius Metellus, Cicero, Claudia Quinta, Clelia, Marcus Curtius, Publius Decius Mure, Marcus Drusus, Quintus Fabius Maximus, Gaius Luscinus Fabritius, Genutius Cipus, Lelius, Gaius Mutius Scevola, Lucius Paulus Emilius, Publius Mucius, Publius Cornelius Scipio, Scipio Emilianus, Scipio Nasica, Manlius Torquatus... ; voir M. CACIORGNA, R. GUERRINI, La virtù figurata. Eroi ed eroine dell'antichità nell'arte senese tra Medioevo e Rinascimento, Sienne, 2003.

66. M. AsCHERI, Siena nel Rinascimento : istituzioni e sistema politico, Sienne, 1985, p. 29, note 36.

67. L'étude classique de N. RubinsteIn, Il governo di Firenze sotto i Medici (1434-1494), Florence, 1971 (traduit en italien à partir de l'édition Oxford 1966) a fait l'objet d'une nouvelle édition, par G. CIAPPELLI, Florence, 1999; pour une analyse et une bibliographie récente, voir A. BRowN, «Smascherare il repubblicanesimo rinascimentale », dans Politica e cultura nelle Repubbliche..., loc. cit., p. 109-133.

68. Consulter H. BUTTERS, "L'ultimo straordinario capitolo del repubblicanesimo fiorentino", dans Politica e cultura nelle Repubbliche..., op. cit., p. 135-155.

69. Voir notamment G. ProcACCI, Machiavelli nella cultura europea dell'età moderna, Rome-Bari, 1995. 70. Le passage (Discorsi I, 55) est commenté avec d'autres dans M. VIROLI, « Il repubblicanesimo di Machiavelli », ibid., p. 157-187.

71. C'est une des considérations les plus connues de ses célèbres Ricordi (II, 110, par exemple dans l'édition de Milan, 1951, p. 95).

72. Voir par exemple, y compris pour les perspectives actuelles, L. BAcCELLI, Critica del repubblicanesimo, Rome-Bari, 2003, ou encore A. CLERICI, Costituzionalismo, contrattualismo e diritto di resistenza nella rivolta dei Paesi Bassi (1559-1581), Milan, 2004.

\section{AUTEUR}

\section{MARIO ASCHER}

Università degli Studi Roma Tre, Facoltà di Giurisprudenza, Via Ostiense 159-161, I-00146 Roma 